\title{
Next-generation X-ray Optics for High Resolution Applications
}

$\underline{\text { Wenbing Yun }}^{1}$, Mark Cordier ${ }^{1}$, Benjamin Stripe ${ }^{1}$, Sylvia Lewis $^{1}$, Janos Kirz $^{1}$

1. Sigray | Concord, CA

wyun@sigray.com

In the past two decades, laboratory x-ray analysis equipment has made significant strides, particularly toward higher resolution capabilities. Although the bottleneck of laboratory x-ray source brightness is well-known and discussed, the need for improvements to develop higher efficiency and higher resolution optics is not as often considered. Without optics with excellent FWHM, ultrahigh brightness x-ray sources cannot be utilized efficiently.

Sigray has made major advances in manufacturing techniques to realize high-grade capillary optics with focusing capabilities down to the submicron scale. These lightweight and compact optics provide the highest flux of any optic, including over $4 \mathrm{X}$ the flux of KB mirrors, and are produced with inner reflecting surfaces of either ellipsoidal or paraboloidal shapes.

Intrinsic and practical advantages of the $\mathrm{x}$-ray optics include:

- $\quad>4 \mathrm{X}$ larger $\mathrm{x}$-ray flux at focus (due to $>2 \mathrm{X}$ larger numerical aperture)

- Potential for $2 X$ higher spatial resolution (intrinsically $4 \mathrm{~nm}$ !)

- Significantly relaxed angular vibration tolerance requirements of upstream beamline optical components, owing to shorter working distance in two orthogonal directions

- Achieving large demagnification of the source and thus small focus due to short working distance

- Substantially larger field of view to achieve high spatial resolution along the X-ray beam axis (e.g., developing super high resolution confocal microXRF)

- Light, low weight and compact form factor allows straightforward scanning of the mirror lens (particularly important when samples are difficult to scan)

- Less complex electro-mechanical system: 4 degrees of freedom instead of 8 or more

- Ease of alignment and to ensure optimal focusing

Sigray has achieved both ellipsoidal and paraboloidal designs that can be used for focusing or collimating $\mathrm{X}$-rays for synchrotron and laboratory use. X-rays are reflected in a single or a double bounce in these optics, which successfully redirect over $80 \%$ of the radiation incident on the inner surface onto the focal spot. Hence such capillaries are highly efficient in preserving the brightness of the source.

In the case of the ellipsoidal design, a portion of an elongated ellipsoid is shaped on the interior surface, so that X-rays originating at one focus of the ellipsoid are reflected to the other focus. Typically, both foci are outside the capillary along the optical axis, providing a finite working distance for positioning the $\mathrm{X}$ ray source and the location where the X-rays are delivered. The numerical aperture of these optics is limited by twice the critical angle of the x-ray energy due to the single-bounce nature of the optic (the deflection angle of the bounce is limited to twice the critical angle).

A single paraboloidal shaped design can be used to focus a parallel incident beam, or to collimate a beam emerging from a focal point so that it emerges as a parallel beam with negligible divergence. Like the ellipsoidal optic, the focal point is outside the capillary, and a beam stop is employed to intercept the portion of the non-reflected beam. For applications requiring point-to-point focusing, a double 
paraboloidal scheme can be used in place of ellipsoidal optics. In this design, a mirrored pair of paraboloidal optics are used. The first capillary renders the beam parallel, while the second brings the parallel beam to the second focus. This twin-paraboloid design involves two reflections, doubling the numerical aperture compared to the single bounce ellipsoid to four times the critical angle.

In all these designs, the focal spot size, which determines the resolution, is typically limited by the accuracy of the optic's surface figure error. Intense and focused research efforts over the past three years have refined a proprietary manufacturing process to achieve accurate and repeatable fabrication. Optical prescription and parameters are chosen to suit the desired application, but in many cases, the optics are hundreds of millimeters long and quite narrow. Because the optical surface is on the interior of the capillary, traditional optical manufacturing and metrology techniques cannot be used to fabricate capillary optics. The small diameter of the optical surface prevents the use of any mechanical grinding and polishing techniques that are typically used when fabricating most mirrors and lenses.

Major process improvements have yielded reliable, sub-micron optics with minimal surface error deviations. To evaluate the optics, Sigray has also developed a ray tracing program to determine the final output of its optics. One example is shown below, using an input source size of 30x60 microns at 22 meters from the source, the angular divergence was limited by the source to sample distance. The source size and divergence in the model were chosen to match the beam parameters at Beamline 3.3.2 at the Advanced Light Source.
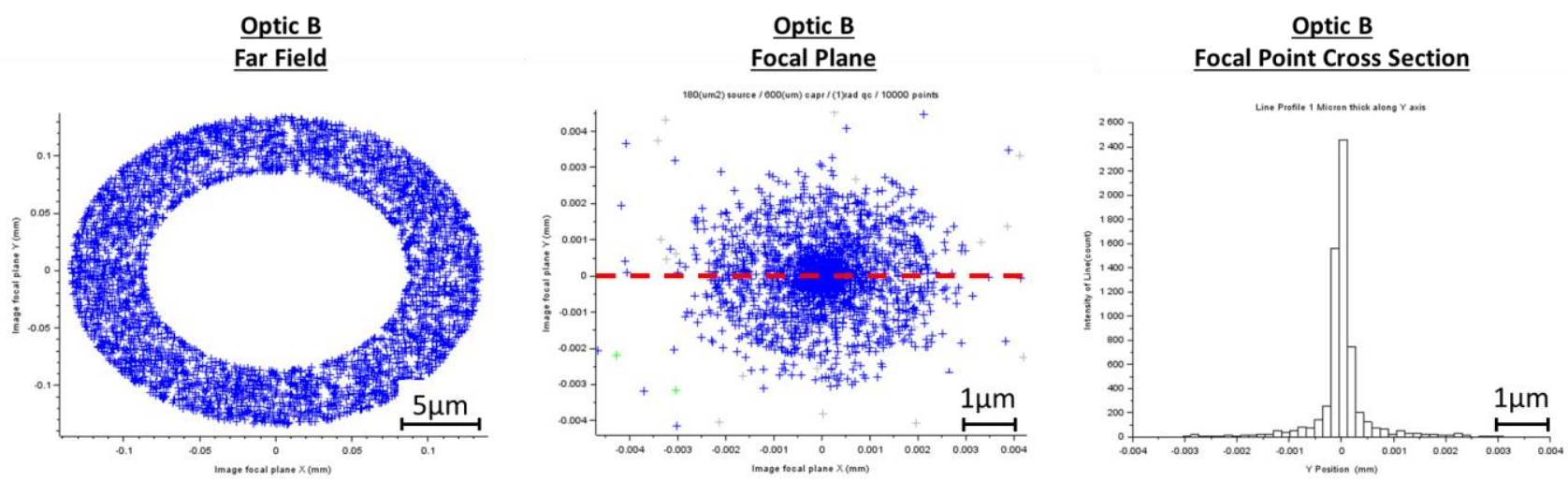

Figure 1. Simulations of the performance of Capillary Optic B, generated using ray tracing software developed at Sigray. Each cross in the blue plots represents a ray striking the plane of interest. The leftmost image is a simulation of the far field pattern generated by this optic, the uniformity of the ring is due to this optic's low surface figure error. The center image is a simulation of the intensity distribution at the focal plane smaller than that of Capillary Optic B. Finally, the rightmost histogram indicated the full width at half maximum of the focal spot is under one micron.

Recent experimental demonstrations of Sigray's optics have demonstrated down to sub-250 nm focusing capabilities in collaboration with researchers from ALS and BNL. Current research focuses include achieving sub-100 nm optics.

Acknowledgments go to the NSF, Division of Industrial Innovation \& Partnerships for funding the development of x-ray mirror lens (IIP-1448727) and to the DOE: Department of Energy (DE-SC0015196) for their small business grants. 